\title{
NOTES ON A PAPER BY SANOV. II
}

RUTH REBEKKA STRUIK

1. Introduction. In this paper another proof is given of some results obtained by Sanov (see [3]).

Let $F=\{u, v\}$ be a free group generated by $u$ and $v$. Let

$$
(x, y)=x^{-1} y^{-1} x y
$$

for $x, y \in F$. If $S, T$ are subgroups of $F$, let

$$
(S, T)=\{(s, t) \mid s \in S, t \in T\} .
$$

Let

$$
F(k)=\left\{x^{k} \mid x \in F\right\} ; \quad F_{1}=F ; \quad F_{k}=\left(F_{k-1}, F\right) .
$$

Let $(u, v, 0)=u,(u, v, 1)=(u, v),(u, v, n)=((u, v, n-1), v)$. Then Sanov [3] proved that

$$
\left(u, v, \alpha p^{\alpha}-1\right)^{p^{\beta-\alpha}} \in F\left(p^{\beta}\right) F_{\alpha p \alpha+1}, \quad \beta, \alpha=1,2, \cdots
$$

where $p$ is a prime.

In this paper, (1.1) is proved for the cases $\alpha=1,2 ; \beta$ arbitrary. A slight generalization of these results is also proved. Sanov's proof involved an investigation of ideals in a Lie Ring. In this paper, Hall's Collection Process will be used. The method also yields other formulas, e.g.

$$
\begin{gathered}
\left(u, v, p^{2}-1\right)^{p^{\beta-1}} \in F_{2 p^{2}-p} F\left(p^{\beta}\right), \\
\left(u, v, p^{\alpha+1}-1\right)^{p^{\beta-1}} \in F_{2 p^{\alpha+1}-p^{\alpha} F\left(p^{\beta}\right), \quad \alpha=1,2, \cdots}
\end{gathered}
$$

and can be used to produce numerous formulas of a similar nature. The author hopes that some of these formulas and/or the method may be of use in solving other group-theoretic problems. The author was unable to use the method to prove (1.1) for $\alpha=3$.

Note that for $\alpha=\beta=1,(1.1)$ becomes

$$
(u, v, p-1) \in F(p) F_{p+1} \text {. }
$$

(1.4) plays an important role in the theory of the Restricted Burnside Problem.

2. Preliminary results. Throughout this paper, $p$ will stand for a prime, and $\alpha, \beta$ for non-negative integers.

Presented to the Society, September 1, 1960; received by the editors August 22, 1960. 
By elementary arguments, the following two lemmas and corollary can be proved:

LEMma 2.1.

$$
C_{p^{\beta}, p^{\alpha}}=p^{\beta-\alpha}(1+s p)
$$

where $s$ is a positive integer. ( $C_{n, r}$ as usual, is the number of combinations of $n$ things taken $r$ at a time.)

Lemma 2.2. $p^{\beta-\alpha} \mid C_{p^{\beta}, s}$ if $p^{\alpha} \leqq s<p^{\alpha+1}$ where $s$ is a positive integer. $(a \mid b$ as usual, means $a$ divides $b$.)

Corollary 2.2. If $s \neq \equiv 0(\bmod p)$, and $p^{\alpha}<s<p^{\alpha+1}$, then $p^{\beta-\alpha+1} \mid C_{p^{\beta}, s .}$ Lemma 2.3. Let $x_{i}, x, i=1,2, \cdots, n$ be $n+1$ elements of a group. Then

$$
\left(\Pi x_{i}, x\right)=\Pi\left(x_{i}, x\right) U
$$

where $U$ is a product of commutators, each of which contains at least 2 of the $x_{i}$ and at least one $x$.

Proof. Use induction on $n$ and the well-known formula

$$
\begin{aligned}
\left(x_{1} x_{2}, x\right) & =\left(x_{1}, x\right)\left(\left(x_{1}, x\right), x_{2}\right)\left(x_{2}, x\right) \\
& =\left(x_{1}, x\right)\left(x_{2}, x\right)\left(\left(x_{1}, x\right), x_{2}\right)\left(\left(\left(x_{1}, x\right), x_{2}\right),\left(x_{2}, x\right)\right) .
\end{aligned}
$$

(See e.g. (10.2.1.2) in [1, p. 150].)

Lemma 2.4 (Hall Collection Formula). Let $u, v$ be any elements of a nilpotent group $F$. Let $p$ be a prime, $\alpha$ any positive integer. Then

$$
(v u)^{p \alpha}=v^{p \alpha} u^{p \alpha} \prod v_{i}^{f_{i}}
$$

where $v_{i}$ is a basic commutator in $u$ and $v$ (see $[1$, p. 178]), and

$$
f_{i}=a_{1} C_{p \alpha, 1}+a_{2} C_{p \alpha, 2}+\cdots+a_{j(i)} C_{p \alpha, j(i)}
$$

where $a_{1}, a_{2}, \cdots, a_{j(i)}$ are non-negative integers and if $v_{i} \in F_{s_{v}}$, then $j(i) \leqq s_{i}$. In particular, if

$$
v_{i}=(u, v, n)
$$

then

$$
f_{i}=C_{p a, n+1}
$$

Proof. See [1, pp. 178-182, 326].

Lemma 2.5. Let $a, b \in F, F$ a nilpotent group. Let $r, s$ be positive integers. Then 


$$
\left(b^{s}, a^{r}\right)=(b, a)^{r s} \prod x_{i}^{C_{r, \lambda} c_{s, \mu}}
$$

where the $x_{i}$ are basic commutators in $b$ and $a$. If $x_{i}$ contains $m_{i} a$ 's and $n_{i} b$ 's, then $\lambda \leqq m_{i}$ and $\mu \leqq n_{i}$. (The exponent of $x_{i}$ will in general be of the form

$$
\sum n_{\lambda \mu} C_{r, \lambda} C_{8, \mu}, n_{\lambda \mu} \text { non-negative integers.) }
$$

Proof. The proof is essentially the same as that given for (2.3) (this paper) [1, pp. 178-182]. Details are given in [5].

Corollary 2.5. Let $b, a$ be as in Lemma 2.5.

$$
\left(b^{p^{\alpha}}, a\right)=(b, a)^{p^{\alpha}} \prod x_{i}^{C_{i}^{\alpha, s_{i j}}}
$$

where if $x_{i}$ is a commutator with $m_{i}$ b's then $s_{i j} \leqq m_{i}$. Each $x_{i}$ contains at least 2 b's.

Definition. $(u, v, n)$ for $n \geqq 1$ is a regular commutator in $u$ and $v$. If $u_{1}$ and $u_{2}$ are regular commutators, so is $\left(u_{1}, u_{2}\right)$.

Comment. $((u, v),(u, v, v))$ is a regular commutator which is not a basic commutator.

LEMmA 2.6. Let $F$ be a nilpotent group. Let $w$ be a regular commutator in $u$ and $v$. Let $w \in F_{s}, k p \leqq s<(k+1) p$. Then either $w$ contains $(k+1) u$ 's or

$$
w^{p^{\beta-1}}=\prod v_{i}^{p^{\beta-1}} \prod_{w_{i}^{p^{\beta-2}}}^{w_{i}} \cdots \prod_{x_{i}^{p^{\beta-t}}}^{w^{\prime}} \cdots\left(\bmod F\left(p^{\beta}\right)\right)
$$

where

$v_{i}, w_{i}, x_{i}$ are regular commutators;

$v_{i} \in F_{s}$ and contains at least $(k+1) u^{\prime} s$;

$w_{i} \in F_{s+\left(p^{2}-p\right)}$ and contains at least as many $u^{\prime} s$ as $w$ does;

$x_{i} \in F_{s+\left(p^{t-p}\right)}$ and contains at least as many $u^{\prime} s$ as $w$ does.

It may be that the $v_{i}^{p \beta-1}, w_{i}^{p \beta-2}, \cdots, x_{i}^{p-t} \cdots$ are scattered among each other.

Proof. Applying Lemmas 2.1, 2.2, Corollary 2.2 to Lemma 2.4 and to equation (2.3), one obtains

$$
\begin{aligned}
(u, v, p-1)^{p^{\beta-1}} & \\
& =\prod v_{i}^{p^{\beta-1}} \prod w_{i}^{p^{\beta-2}} \cdots \prod x_{i}^{p^{\beta-t}} \cdots\left(\bmod F\left(p^{\beta}\right)\right)
\end{aligned}
$$

where $v_{i}, w_{i}, x_{i}$ are regular commutators; $v_{i} \in F_{p}$ and contains at least $2 u$ 's unless it is of the form $(u, v, n p-1) n>1 ; w_{i} \in F_{p^{2}}, x_{i} \in F_{p^{t}}$.

Replace $u$ by $(u, v,(n-1) p)$ on each side of (2.10). This gives 


$$
\begin{aligned}
(u, v, n p-1)^{p^{\beta-1}} & \\
& =\prod y_{i}^{p^{\beta-1}} \prod z_{i}^{p^{\beta-2}} \cdots \prod u_{i}^{p^{\beta-t}} \cdots\left(\bmod F\left(p^{\beta}\right)\right)
\end{aligned}
$$

where $y_{i} \in F_{n p}$ and contains $2 u^{\prime}$ 's unless it is of the form

$$
(u, v, m p-1) m>n ; z_{i} \in F_{p^{2}+n p}, u_{i} \in F_{p^{t}+n p} ;
$$

$y_{i}, z_{i}, u_{i}$ are regular commutators and may be scattered among each other. Replace the right hand side of $(2.11)$ wherever $(u, v, n p-1)^{p^{\beta-1}}$ appears on the right hand side of (2.10). Repeat as often as needed until Lemma 2.6 is proved for $s=p$ and $k=1$. This gives

$$
\begin{aligned}
(u, v, p-1)^{p^{\beta-1}} & \\
& =\prod v_{i}^{p^{\beta-1}} \prod w_{i}^{p^{\beta-2}} \cdots \prod x_{i}^{p^{\beta-t}} \cdots\left(\bmod F\left(p^{\beta}\right)\right)
\end{aligned}
$$

where

$v_{i}, w_{i}, x_{i}$ are regular commutators in $u$ and $v$;

$v_{i} \in F_{p}$ and contains at least $2 u$ 's;

$w_{i} \in F_{p^{2}}, x_{i} \in F_{p^{t}}$.

Note that the $v_{i}, w_{i}, x_{i}$ of (2.12) are different from those of (2.10). Using the same letters to designate different commutators will be done frequently in the subsequent argument to maintain uniformity of notation.

Replace $u$ by $(u, v, n)$ on both sides of (2.12). This proves (2.9) for $k=1, s=p, p+1, \cdots, 2 p-1$. Assume true for $s<r$. Then if $w \in F_{r}$, $k p \leqq r \leqq(k+1) p$ and does not contain $(k+1) u$ 's, then one of its components is $(u, v, n), n \geqq p-1$. Then if $w=(b, a)$, where $a, b$ are regular commutators, and $b$ contains $(u, v, n)$,

$$
(b, a)^{p^{\beta-1}}=\left(b^{p^{\beta-1}}, a\right) \prod y_{i}^{C_{p}^{\beta-1}, s_{i j}}\left(\bmod F\left(p^{\beta}\right)\right)
$$

where (2.8) has been used with $\alpha$ replaced by $\beta-1, y_{i}$ regular commutators. Using induction (on $\left(b^{p^{\beta-1}}, a\right)$ ), Lemma 2.3 and Corollary 2.5 repeatedly, one can eventually put $w^{p^{\beta-1}}$ into the desired form.

Comment. If we replace $u$ by $(u, v, n)$ on both sides of (2.12) we obtain

$$
\begin{aligned}
(u, v, p- & 1+n)^{p^{\beta-1}} \\
& =\prod v_{i}^{p^{\beta-1}} \prod w_{i}^{p^{\beta-2}} \cdots \Pi x_{i}^{p^{\beta-t}} \cdots\left(\bmod F\left(p^{\beta}\right)\right)
\end{aligned}
$$

where $v_{i}, w_{i}, x_{i}$, are regular commutators (but not the same $v_{i}, w_{i}, x_{i}$ as in (2.12)), $v_{i} \in F_{p+2 n}$ and contains at least two $u$ 's, $w_{i} \in F_{p^{2}+n}$, $x_{i} \in F_{p^{t}+n}$.

Let $p+2 n=p^{2}+n$, then $n=p^{2}-p$. This gives 


$$
\left(u, v, p^{2}-1\right)^{p^{\beta-1}}=\prod w_{i}^{p^{\beta-2}} \cdots \prod x_{i}^{p^{\beta-t}} \cdots\left(\bmod F\left(p^{\beta}\right)\right)
$$

where $w_{i}, x_{i}$ are regular commutators, $w_{i} \in F_{2 p^{2}-p}, x_{i} \in F_{p^{t}+p^{2}-p}$ which proves (1.2) and is a slight generalization of (1.2).

\section{Theorems.}

THEOREM 3.1 .

$$
(u, v, p-1)^{p^{\beta-1}} \in F\left(p^{\beta}\right) F_{p+1} .
$$

Proof. The proof for the case $\beta=1$ given in $[1$, p. 327] goes through, mutatis mutandis, replacing $p^{\beta}$ by $p$. Equation (2.10) of this paper plays the role of (18.4.9) in [1, p. 327].

If one uses (2.12), the above proof for (3.1), the fact that any commutator can be written as a product of basic (or regular commutators), and Lemma 2.4, and the device used in going from (2.10) to (2.12), one obtains,

$$
\begin{array}{r}
(u, v, p-1)^{p^{\beta-1}} \\
\quad=\prod v_{i}^{p^{\beta-1}} \prod w_{i}^{p^{\beta-2}} \cdots \prod x_{i}^{p^{\beta-t}} \cdots\left(\bmod F\left(p^{\beta}\right)\right)
\end{array}
$$

where $v_{i} \in F_{p+1}$ and contains at least $2 u^{\prime} s, w_{i} \in F_{p^{2}}, x_{i} \in F_{p^{l}} ; v_{i}, w_{i}, x_{i}$ regular commutators. (The $v_{i}, w_{i}, x_{i}$ may be scattered among each other.)

(3.2) is a slight generalization of (3.1).

\section{Theorem 3.2.}

$$
\left(u, v 2 p^{2}-1\right)^{p^{\beta-2}} \in F\left(p^{\beta}\right) F_{2 p^{2}+1} .
$$

Proof. From Lemma 2.4, and using Lemmas 2.1, 2.2 and Corollary 2.2, one obtains

$$
\begin{aligned}
\left(u, v, p^{2}-1\right)^{p^{\beta-2}} & \\
= & \prod v_{i}^{p^{\beta-1}} \prod w_{i}^{p^{\beta-2}} \cdots \prod x_{i}^{p^{\beta-t}} \cdots\left(\bmod F\left(p^{\beta}\right)\right)
\end{aligned}
$$

where $v_{i}, w_{i}, x_{i}$ are regular commutators, $v_{i} \in F_{p}, x_{i} \in F_{p^{t}}, w_{i} \in F_{p^{2}}$, and if $w_{i} \notin F_{p^{2}+1}$ it contains at least $2 u$ 's; $v_{i}$ contains at least $2 u^{\prime}$ s unless it is of the form $(u, v, n p-1), n \geqq 1$. Replace $u$ by $\left(u, v, p^{2}-p\right)$ on both sides of (3.4):

$$
\begin{aligned}
\left(u, v, 2 p^{2}-p-1\right)^{p^{\beta-2}} & \\
& =\prod v_{i}^{p^{\beta-1}} \prod w_{i}^{p^{\beta-2}} \cdots \prod x_{i}^{p^{\beta-t}} \cdots\left(\bmod F\left(p^{\beta}\right)\right)
\end{aligned}
$$

$v_{i}, w_{i}, x_{i}$ are regular commutators, $v_{i} \in F_{p^{2}}, w_{i} \in F_{2 p^{2}-p}$; if $w_{i} \notin F_{2 p^{2}-p+1}$ it contains at least $2 u^{\prime}$ s, $x_{i} \in F_{p^{t}+\left(p^{2}-p\right)}$. 
Using Lemma 2.4 with $\alpha=\beta+1$, one obtains

$$
\left(u, v, p^{2}-1\right)^{p^{\beta-1}}=\prod v_{i}^{p^{\beta-1}} \prod w_{i}^{p^{\beta-2}} \cdots\left(\bmod F\left(p^{\beta}\right)\right)
$$

where $v_{i}, w_{i}$ are basic commutators, $v_{i} \in F_{p^{2}}$ and if $v_{i} \neq\left(u, v, p^{2}-n p-1\right)$, it contains at least $2 u^{\prime} s, w_{i} \in F_{p^{3}}$. Use the same device that obtained (2.12) from (2.10): this means that one can assume in (3.6) that all $v_{i}$ contain at least two $u$ 's. Replace $u$ by $(u, v, n)$ on both sides of (3.6):

$$
\left(u, v, p^{2}-1+n\right)^{p^{\beta-1}}=\prod v_{i}^{p^{\beta-1}} \prod w_{i}^{p^{\beta-2}} \cdots\left(\bmod F\left(p^{\beta}\right)\right)
$$

where $v_{i} \in F_{p^{2}+2 n}, w_{i} \in F_{p^{3+n}+n} ; v_{i}, w_{i}$ are regular commutators and may be scattered among each other.

Put the right hand side of (3.7) wherever $\left(u, v, p^{2}-1+n\right)^{p^{\beta-1}}$ appears on the right hand side of (3.5). This means one can assume that all $v_{i}$ contain at least two $u$ 's. Now apply Lemma 2.6 with $k=p$. This means one can assume each $v_{i}$ contains at least $(p+1) u$ 's. Replace $u$ by $(u, v, p)$ on both sides of (3.5). This gives

$$
\left(u, v, 2 p^{2}-1\right)^{p^{\beta-2}}=\prod w_{i}^{p^{\beta-2}} \cdots \prod x_{i}^{p^{\beta-t}} \cdots\left(\bmod F\left(p^{\beta}\right)\right),
$$

where $w_{i}, x_{i}$ are regular commutators, $w_{i} \in F_{2 p^{2}+1}, x_{i} \in F_{p^{t}+p^{2}}$. This is sufficient to prove (3.3). Note that by using the same device used in going from (2.10) to (2.12), one can assume that each $w_{i}$ of (3.8) contains at least two $u$ 's. (3.8) is a slight generalization of Theorem 3.2.

Starting with (3.6) and using the same device used in going from (2.12) to $(2.14)$ to $(2.15)$, one obtains

$$
\begin{aligned}
\left(u, v, p^{3}-1\right)^{p^{\beta-1}} & \\
= & \prod v_{i}^{p^{\beta-2}} \prod w_{i}^{p^{\beta-3}} \cdots \prod_{i}^{p^{\beta-t}} \cdots\left(\bmod F\left(p^{\beta}\right)\right)
\end{aligned}
$$

where $v_{i}, w_{i}, x_{i}$ are regular commutators (which may be scattered among each other), $v_{i} \in F_{2 p^{3}-p^{2}}, w_{i} \in F_{p^{4}+\left(p^{3}-p^{2}\right)}, x_{i} \in F_{p^{t}+\left(p^{8}-p^{2}\right)}$ which gives (1.3), for $\alpha=2$. (1.3) can be proved similarly.

\section{BIBLIOGRAPHY}

1. M. Hall, Jr., The theory of groups, New York, Macmillan, 1959.

2. O. Ore, Number theory and its history, New York, McGraw-Hill, 1948.

3. I. N. Sanov, On a certain system of relations in periodic groups with period a power of a prime number, Izv. Akad. Nauk SSSR. Ser. Mat. vol. 15 (1951) pp. 477-502.

4. R. R. Struik, Notes on a paper by Sanov, Proc. Amer. Math. Soc. vol. 8 (1957) pp. 638-641.

5. - On nilpotent products of cyclic groups. II, Canad. J. Math., to appear.

University of British Columbia 